\title{
Intoxicación familiar por mercurio elemental. Caso clínico
}

\author{
JAIME VALDERAS J. ${ }^{1,2}$, MARÍA EMILIA MEJÍAS P. ${ }^{1}$, JOEL RIQUELME R. ${ }^{2}$, \\ KARINA AEDO S. ${ }^{1}$, SOFÍA AROS A. ${ }^{1,2}$, FRANCISCO BARRERA Q. ${ }^{2}$ \\ 1. Departamento de Pediatría y Cirugía Infantil Campus Centro, Facultad de Medicina, Universidad de Chile. \\ 2. Unidad de Agudos, Servicio de Pediatría, Hospital Clínico San Borja Arriarán.
}

\begin{abstract}
Family poisoned by elemental mercury: a case report

Introduction: Mercury is a heavy metal widely dispersed in nature, occurring in three chemical forms. Exposure to mercury at work sites and even at home may be clinically significant. Objective: To update the knowledge about the risks of this toxic element. Case report: the case of a teenager and his family poisoned by elemental mercury is reported. The diagnostic process was difficult, mainly due to an initial presumption of probable infectious etiology, unavailability of key anamnestic data and unusual clinical behavior, with signs and symptoms of multisystem compromise (neurological, hepatic, renal and dermatological compromise). Discussion: The study was based on literature review of various clinical presentations regarding this poisoning and its management, emphasizing the need for dimercaptosuccinic acid chelator. As a major public health problem, the importance of education and implementation of public policies to have a mercury-free environment is discussed.
\end{abstract}

(Key words: Mercury, elemental mercury, mercury poisoning, exposure to elemental mercury, dimercaptosuccinic acid).

Rev Chil Pediatr 2013; 84 (1): 72-79

\section{RESUMEN}

Introducción: El mercurio es un metal pesado ampliamente distribuido en el medio ambiente, en sus tres formas químicas. La exposición a dicho metal en recintos laborales e incluso en el hogar, puede llegar a ser clínicamente significativa. Objetivo: Actualizar el conocimiento acerca de los riesgos de este tóxico. Caso clínico: Se presenta el caso clínico de un adolescente y su familia intoxicados por mercurio elemental, cuyo proceso diagnóstico fue difícil, principalmente por la presunción inicial de una probable etiología infecciosa, falta de disponibilidad de datos anamnésticos claves y el inusual comportamiento clínico, con signos y síntomas de compromiso multisistémico (neurológico, hepático, renal y dermatológico). Discusión: Se revisa la literatura en relación a las diversas formas de presentación clínica de esta intoxicación y su manejo, destacando

Recibido el 12 de abril de 2012, devuelto para corregir el 04 de julio de 2012, segunda versión el 17 de agosto de 2012, aceptado para publicación el 28 de septiembre de 2012.

Este trabajo cumple con los requisitos sobre consentimiento/asentimiento informado, comité de ética, financiamiento, estudios animales y sobre la ausencia de conflictos de intereses según corresponda.

Correspondencia a:

Francisco Barrera Q.

E-mail: fbarreraq@gmail.com 
la utilidad del quelante ácido dimercaptosuccínico. Por ser un importante problema de salud pública, se destaca la trascendencia de la educación e implementación de políticas públicas por un ambiente libre de mercurio.

(Palabras clave: Mercurio, mercurio elemental, intoxicación por mercurio, exposición a mercurio elemental, ácido dimercaptosuccínico).

Rev Chil Pediatr 2013; 84 (1): 72-79

\section{Introducción}

Mientras la exposición a niveles tóxicos de cualquier contaminante ambiental puede resultar nociva para el adulto, los efectos son más deletéreos para los sistemas en desarrollo del niño, en especial el sistema nervioso central. Los metales están entre los tóxicos más antiguos descritos por el hombre. En el industrializado mundo actual, las fuentes de exposición a metales son múltiples, tanto en el campo laboral como a partir de agua, alimentos o ambiente contaminados. Su toxicidad está caracterizada por el elemento metálico en cuestión, pero se ve modificada por el tipo de compuesto, orgánico o inorgánico y sus características de hidro o liposolubilidad, que determinan su toxicocinética. Las biomoléculas más afectadas por los metales son las proteínas con actividad enzimática por lo que su compromiso es multisistémico (gastrointestinal, neurológico central y periférico, hematológico y renal, entre otros).

El mercurio $(\mathrm{Hg})$ es un metal pesado ubicuo en todo el planeta. Proviene de fuentes naturales y artificiales. Existen 3 principales formas químicas del mercurio, todas tóxicas pero con diferentes vías de absorción, manifestaciones clínicas y respuesta a terapia, a saber: mercurio metálico o elemental $\left(\mathrm{Hg}^{\circ}\right)$, mercurio inorgánico o sales de mercurio $\left(\mathrm{Hg}^{+1}, \mathrm{Hg}^{+2}\right)$ y mercurio orgánico ${ }^{1}$ (tabla 1). El mercurio elemental es blanco plateado brillante, líquido a temperatura ambiente, con propiedades físicas y químicas únicas. El mercurio orgánico, en forma de metilmercurio en peces y mariscos, o de etilmercurio o timerosal, es la forma más peligrosa para la salud humana ${ }^{2}$.

El mercurio se encuentra presente en termómetros, amalgamas, preservantes de vacunas, peces y en la atmósfera. La liberación antropogénica ambiental de $\mathrm{Hg}$ se calcula en 2.000 toneladas al año ${ }^{3}$. Las principales fuentes de contaminantes mercuriales han sido la actividad minera, residuos industriales de plantas cloro-alcali o de fabricación de vinilo y fungicidas, pinturas antifúngicas, fotografía, pirotecnia, baterías secas y pilas, industrias papeleras y laboratorios médico-veterinarios y dentales.

La exposición aguda o crónica puede cau-

Tabla 1. Características de las diferentes formas químicas del Hg

\begin{tabular}{|c|c|c|c|c|}
\hline Mercurio & Fuente & Ruta de exposición & Eliminación & Toxicidad \\
\hline Metálico o elemental & $\begin{array}{l}\text { Amalgamas dentales } \\
\text { Orfebrería artesanal } \\
\text { Termómetro } \\
\text { Esfigmomanómetro } \\
\text { Remedios folclóricos } \\
\text { Erupciones volcánicas }\end{array}$ & Inhalatoria & $\begin{array}{l}\text { Orina } \\
\text { Heces }\end{array}$ & $\begin{array}{l}\text { SNC } \\
\text { Renal } \\
\text { Piel } \\
\text { Pulmonar }\end{array}$ \\
\hline Inorgánico o sales de mercurio & $\begin{array}{l}\text { Timerosal } \\
\text { Cosméticos } \\
\text { Ampolletas } \\
\text { Productos fotográfico } \\
\text { Desinfectantes }\end{array}$ & $\begin{array}{l}\text { Digestiva } \\
\text { Cutánea }\end{array}$ & Orina & $\begin{array}{l}\text { SNC } \\
\text { Renal } \\
\text { Piel } \\
\text { Pulmonar }\end{array}$ \\
\hline Orgánico o metil mercurio & $\begin{array}{l}\text { Pescados } \\
\text { Preservantes } \\
\text { Fungicidas }\end{array}$ & $\begin{array}{l}\text { Digestiva } \\
\text { Transplacentaria } \\
\text { Parenteral }\end{array}$ & Heces & $\begin{array}{l}\text { SNC } \\
\text { Cardiovascular }\end{array}$ \\
\hline
\end{tabular}


sar efectos adversos durante cualquier período del desarrollo. No existe un nivel conocido de exposición que sea seguro, más aún, se sabe que el ser humano no debiera tener mercurio en su organismo ya que éste no tiene funciones fisiológicas demostradas. Es altamente tóxico debido a su gran afinidad por grupos sulfhidrilos presentes en proteínas con actividad enzimática, con funciones de transporte y estructurales que se expresan en diferentes tejidos. Es un importante problema de salud ambiental y si bien los efectos deletéreos en la salud estaban descritos hace siglos por Hipócrates y Galeno, las masivas intoxicaciones con metilmercurio en Japón y en Irak, han sido la alerta de los tiempos modernos. En 1956, en la Bahía de Minamata (pueblo pesquero de la isla de Kyushu, Japón), se produjo la muerte de 46 personas, aunque si se considera todo el período de daño entre 1953 y 1965, se informó oficialmente de 111 víctimas fatales y más de 400 casos con problemas neurológicos graves. Incluso madres asintomáticas dieron a luz recién nacidos gravemente afectados. En 1968, el gobierno japonés anunció oficialmente que la causa de la enfermedad era la ingestión de pescados y mariscos contaminados con metil mercurio, derivado de la degradación bacteriana del mercurio vertido a las aguas por la empresa Petroquímica Chaisso (en total 81 toneladas de mercurio $)^{4}$. Estudios posteriores informaron de mucha mayor morbimortalidad. Dicha patología se denomina hoy como enfermedad de Minamata. En Chile y en todo el mundo continúan produciéndose tragedias ecológicas, algunas de ellas con mucho ruido y otras con silenciosa complicidad.

En USA y Europa existen políticas por un ambiente libre de mercurio y recomendaciones como dosis de referencia (RfD) para Hg orgánico (cantidad ingerida en un período de tiempo sin producir efectos adversos para la salud). La Environmental Protection Agency recomienda RfD de $0,1 \mathrm{mcg} / \mathrm{kg} / \mathrm{d}$ o de $5,8 \mathrm{mcg} / \mathrm{l}$ en sangre de cordón. En especial se recomienda a embarazadas y niños no ingerir peces de gran tamaño que consumen otros peces (blanquillo, caballa, atún) por el efecto de biomagnificación por la cadena alimenticia. Por otro lado, se ha asociado el timerosal de las vacunas con alteraciones del desarrollo, especialmente autismo y aunque no hay estudios poblacionales que lo demuestren, por el eventual riesgo, se ha suspendido su uso en muchos países ${ }^{1}$. El objetivo del presente artículo es comunicar el caso de una familia intoxicada por mercurio elemental y a partir de ello, alertar al equipo de salud los riesgos de este metal pesado y revisar la literatura disponible.

\section{Caso clínico}

Paciente EVM 13 años sin antecedentes mórbidos nacido en Bolivia, viviendo en Chile desde 2009. En junio de 2011 inicia fiebre hasta $40^{\circ} \mathrm{C}$ y exantema micropapular eritematoso de distribución generalizada con compromiso palmo plantar, diagnosticándose escarlatina. Madre (37 años), hermana (12 años) y hermano (10 años) presentan cuadro clínico similar. Todos reciben tratamiento con penicilina benzatina intramuscular y amoxicilina oral por 7 días. EVM se mantiene con fiebre y exantema (figuras 1 y 2), agregándose deposiciones disentéricas. Presenta edema de pies y manos, persiste febril, se constata transaminasas elevadas, leucopenia y se decide hospitalizar. Madre evoluciona con cuadro autolimitado al igual que hermano menor, destacando hipocomplementemia e hiper IgG que persiste aún resuelta su sintomatología. Hermana evoluciona con edema facial, hipertransaminasemia moderada (hasta $600 \mathrm{UI} / 1$ ), proteinuria masiva (hasta $200 \mathrm{mg} / \mathrm{m}^{2} /$ hora), hipocomplementemia persistente, se diagnostica síndrome nefrótico.

EVM presenta una mala evolución en las primeras semanas, con compromiso multisistémico progresivo. Se realiza estudio que incluye causas infecciosas, inmunológicas, metabólicas y alérgicas. La anamnesis dirigida no permite identificar exposición a tóxicos. Evoluciona con mayor decaimiento, anorexia, letargia, baja de peso $(9 \%)$, con compromiso de conciencia cualitativo y oscilante. Presenta sintomatología y laboratorio compatible con falla hepática aguda y se traslada a Centro Especializado para eventual trasplante hepático. Se plantea Síndrome DRESS (drug reaction with eosinophilia and systemic symptoms), iniciándose 


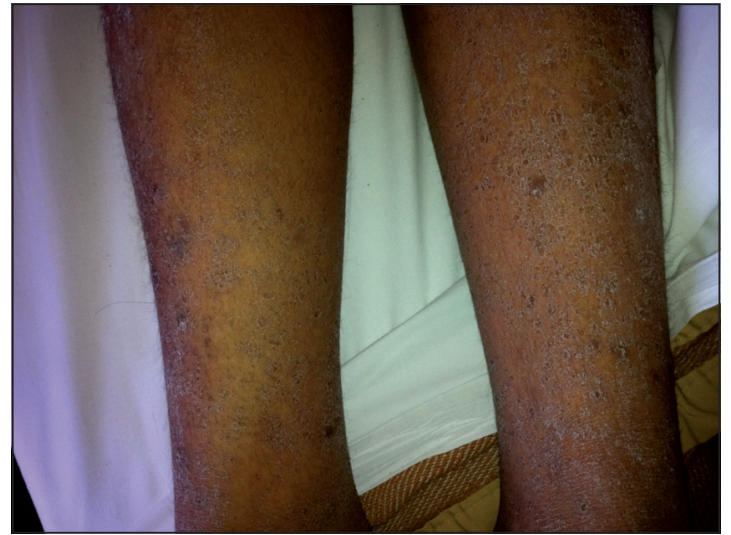

Figura 1. Paciente EVM. Lesiones eritemato descamativas en extremidades inferiores.

tratamiento con metilprednisolona, que recibe por 6 días. Evoluciona con mejoría clínica y de laboratorio, por lo que regresa a hospital de origen. Ante recuperación gradual de todos los síntomas, se decide alta. Se logra completar estudio etiológico para metales pesados, con determinación de niveles de mercurio en orina en Instituto de Salud Pública, que resultan elevados. Los niveles de mercurio en orina de la madre y hermanos también se encuentran en rango de intoxicación (tabla 2 ).

Los 4 integrantes de la familia recibieron tratamiento quelante con Ácido Dimercaptosuccínico por vía oral por 19 días y Sulfato de zinc vía oral. Actualmente EVM asintomático con compromiso hepático resuelto, madre

Tabla 2. Niveles Urinarios de Hg inorgánico (pre y post tratamiento) en familia afectada

\begin{tabular}{|c|c|c|}
\hline \multirow[t]{2}{*}{ Paciente } & \multicolumn{2}{|c|}{$\begin{array}{l}\text { Niveles Urinarios } \\
\text { Hg Inorgánico* }\end{array}$} \\
\hline & $\begin{array}{c}\text { Pre } \\
\text { tratamiento }\end{array}$ & $\begin{array}{c}\text { Post } \\
\text { tratamiento }\end{array}$ \\
\hline EVM (13 años) & 287 ug/g Creat & 198 ug/g Creat \\
\hline Hermano (10 años) & 1.291 ug/g Creat & 321 ug/g Creat \\
\hline Hermana (12 años) & 152 ug/g Creat & 155 ug/g Creat \\
\hline Madre (47 años) & 534 ug/g Creat & 139 ug/g Creat \\
\hline
\end{tabular}

*Técnica: Mercurio, Espectrofotometría de absorción atómica, generación de hidruros; creatinina, espectrofotometría UV-Vis. Según niveles de creatinina en orina los valores de mercurio pueden expresarse en ug/L o ug/g. Valor normal: $<10 \mathrm{ug} / \mathrm{L}$ ó $<50 \mathrm{ug} / \mathrm{g}$ creatininuria. ${ }^{\ddagger}$ Tratamiento realizado con Acido Dimercaptosuccínico vía oral por 19 días. Control post tratamiento se realizó 3 semanas después.

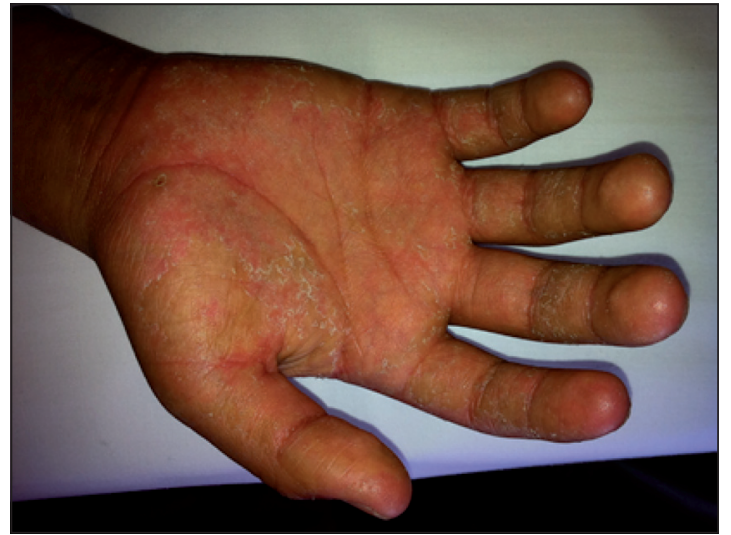

Figura 2. Paciente EVM. Acrodinia.

asintomática sin evidencia de daño en ningún parénquima y hermanos con proteinuria moderada. Los niveles urinarios de $\mathrm{Hg}$ de control 3 semanas después del tratamiento disminuyeron significativamente pero persistieron sobre rango aceptable en 2 pacientes, requiriendo nuevo tratamiento. Todos se encuentran en seguimiento ambulatorio.

En las indagaciones acerca del origen del mercurio elemental fue imposible determinar de donde provenía, pero pudo precisarse que fue encontrado en la vía pública, en las cercanías del colegio de EVM y de una feria pública del sector, en un frasco plástico con contenido líquido metálico. Este $\mathrm{Hg}$ (aproximadamente $40 \mathrm{ml}$ ), es llevado por los menores al recinto educacional y parte de éste al domicilio de la familia, catorce días previos al inicio de los síntomas. Allí es manipulado por los hermanos y su madre, y finalmente derramado en el único dormitorio de la familia. La madre retira parcialmente estos restos con un paño de aseo. Se notificó de todo lo ocurrido y de los riesgos a la autoridad de salud y de educación.

\section{Discusión}

El caso clínico presentado grafica la evolución clínica y tratamiento de una intoxicación familiar por vapores de mercurio elemental (metálico). Esta forma química del $\mathrm{Hg}$ resulta muy atractiva para los niños debido a su color plateado, densidad y tendencia a formar cuentas. 
En la práctica, la intoxicación por mercurio elemental puede tener múltiples fuentes como la inhalación de vapores, exposición a polvos, pinturas o personas laboralmente contaminadas con mercurio, ingestión accidental de instrumentos y amalgamas, exposición laboral en minas de oro (África, Asia y América Latina). En domicilio se encuentra principalmente en termómetros, barómetros, ampolletas fluorescentes compactas, interruptores eléctricos, reguladores de gas natural. En las escuelas puede encontrarse mercurio almacenado en laboratorios de ciencias y piso de poliuretano en gimnasios ${ }^{1}$.

Lee et al., describe 26 eventos de intoxicación, la mayoría (56\%) en la casa y $42 \%$ en el colegio, el resto en centros de salud, propiedad industrial, jardín infantil y automóvil. La exposición al mercurio de un termómetro quebrado fue el escenario más común y en la gran mayoría de los casos fue un error humano por inapropiada manipulación o mala limpieza del mercurio derramado ${ }^{6}$. En el caso particular de la familia afectada, los niños estuvieron jugando con el metal por varios días en su casa. Finalmente el elemento fue derramado sobre el piso $\mathrm{y}$, a pesar que la madre procuró hacer un aseo prolijo de la habitación, probablemente quedó $\mathrm{Hg}$ residual, cuyos vapores fueron inhalados en forma sostenida, hasta hacerse sintomáticos.

La absorción del mercurio metálico o elemental se efectúa principalmente a través de inhalación de sus vapores, con una penetración a través de la membrana alvéolo-capilar de hasta el 75\% de la dosis inhalada. La absorción por vía digestiva y piel es despreciable. En la sangre difunde al interior de los glóbulos rojos donde es oxidado a ión mercúrico, al igual que en los tejidos, por catalasas peroxisomales, en un proceso que es reversible. El Hg no oxidado es capaz de penetrar a través de la barrera hematoencefálica y la placenta. En el SNC queda atrapado en forma de ión mercúrico, por lo que el daño a este nivel es sostenido en el tiempo. De esta forma el mercurio metálico se distribuye por la sangre y se acumula en altas concentraciones en el cerebro y los riñones. $\mathrm{La}$ piel, el pelo, el hígado, las glándulas salivales, los testículos y el intestino, muestran también presencia de mercurio, pero en menor cantidad. Su eliminación es urinaria y digestiva en forma de ión mercúrico, y su vida media en el organismo es de hasta 60 días ${ }^{6,7}$.

La intoxicación aguda por inhalación de $\mathrm{Hg}$ elemental se caracteriza por síntomas iniciales respiratorios como disnea, tos seca asociada a fiebre y calofríos. El cuadro puede evolucionar hacia una neumonitis intersticial con atelectasias y enfisema, e incluso a un síndrome de dificultad respiratoria del adulto, causa de mortalidad en intoxicación aguda por $\mathrm{Hg}$. Se acompaña además de síntomas digestivos inespecíficos como náuseas, vómitos y diarrea, sabor metálico, sialorrea, disfagia y gingivoestomatitis. Pueden aparecer síntomas neurológicos y alteraciones visuales, así como también insuficiencia renal (por necrosis tubular aguda), todo ello como expresión de la conversión tisular a ión mercúrico. En ocasiones es posible también apreciar un compromiso cutáneo denominado acrodinia, caracterizado por una induración hiperqueratósica de la cara con edema e inflamación palmo plantar, rash eritematoso, descamación e hiperhidrosis. Se atribuye a una reacción de hipersensibilidad idiosincrática ${ }^{7}$.

La intoxicación subaguda o crónica, es una forma de presentación clínica más frecuente que la aguda y afecta en todos los casos al sistema nervioso central, además de los órganos ya mencionados. Aparece a lo largo de semanas, meses o años, y se caracteriza por una alteración de mucosas (estomatitis y gingivitis que puede llevar a la pérdida de piezas dentarias) además de un temblor involuntario que se inicia en los dedos de manos, párpados, labios y lengua, progresando posteriormente a las extremidades, desaparece durante el sueño y aumenta con estados de excitación. Otras manifestaciones neurológicas descritas son la polineuropatía sensitivo motora, anosmia, disminución del campo visual y ataxia ${ }^{2}$. El cuadro clínico subagudo y crónico suele también acompañarse de trastornos conductuales y del ánimo, como depresión, crisis de llanto inmotivado, ansiedad, irritabilidad, violencia, amnesia parcial, insomnio, alucinaciones y psicosis maniaco-depresiva. Este cuadro clínico se denomina eretismo mercurial. La intoxicación 
crónica puede cursar con alteraciones renales que van desde la proteinuria aislada al síndrome nefrótico, en el contexto de nefropatía membranosa secundaria a nefritis por inmunocomplejos (depósitos de Ig G1 y de $\left.\mathrm{C}_{3}\right)^{8-10}$.

Puede presentarse, también, compromiso hepático. Aunque éste es infrecuente, puede ir desde hepatitis hasta falla hepática fulminante. Una publicación reciente reporta un caso de intoxicación por mercurio, falla hepática y síndrome DRESS. Si bien la etiopatogenia del compromiso hepático no está del todo clara, podrían estar involucrados mecanismos auto inmunes (hipersensibilidad retardada tipo IV) ${ }^{11}$.

Similar a lo descrito en la literatura, los integrantes de esta familia intoxicados con $\mathrm{Hg}$ elemental, en particular EVM, presentaron un amplio espectro de signos y síntomas, tales como fiebre, compromiso cutáneo (acrodinia), renal (síndrome nefrótico), compromiso neurológico (encefalopatía), trastornos del ánimo y compromiso hepático (hepatitis, ictericia colestásica e insuficiencia hepática). Cabe destacar que existen escasos reportes en la literatura internacional de compromiso de función hepática secundario a intoxicación mercurial en niños, por lo que el presente caso contribuye a generar conocimiento respecto al comportamiento clínico de esta patología en pediatría.

El compromiso multisistémico de la intoxicación por $\mathrm{Hg}$ elemental pone de manifiesto la necesidad del conocimiento de esta entidad nosológica a fin de tener un adecuado índice de sospecha que permita llegar a plantear el diagnóstico, después de una exhaustiva y completa anamnesis y examen físico. Nuestro planteamiento etiológico inicial y de manejo en estos pacientes fue el infeccioso, que al ser descartado, hizo necesario plantear otras etiologías multisistémicas, incluyendo la intoxicación por metales pesados.

El tratamiento de la intoxicación por mercurio metálico se basa fundamentalmente en el manejo ambiental o erradicación de la fuente de contaminación y el tratamiento quelante ${ }^{12}$ :

1. Manejo ambiental: En Europa y Estados Unidos existen guías clínicas que abordan el manejo extra-hospitalario de la intoxicación por mercurio metálico. En Chile, la Secretaría Ministerial de Salud (SEREMI) respectiva, debe tomar las medidas necesarias para el manejo ambiental ante un derrame de este metal. La recomendación internacional se basa en la cantidad aproximada de mercurio derramado; si este corresponde a la cantidad contenida en un termómetro clínico $(0,5$ a $0,7 \mathrm{~g})$, sólo bastaría con recoger dicho metal, introducirlo en una bolsa plástica, eliminándola en contenedores para este efecto ubicados en Centros de Salud que tengan habilitadas bodegas de REAS (Residuos de Establecimientos de Atención de Salud) y ventilar la habitación afectada. Si la cantidad es mayor a la contenida en un termómetro clínico y menor a $30 \mathrm{ml}$, se deben tomar las mismas medidas anteriores y notificar a la SEREMI de salud correspondiente. Si la cantidad derramada es mayor de $30 \mathrm{ml}$ (el mercurio contenido en un esfigmomanómetro) se debe abandonar el lugar y dar aviso a la autoridad competente. Para la recolección del metal derramado se debe tener presente que el método a usar no debe aumentar la volatilidad del metal, por lo tanto, nunca se debe aspirar, solo recoger para luego eliminar los instrumentos en bolsas plásticas, incluida la ropa y calzados usados $^{12}$.

2. Tratamiento quelante: Un quelante es una molécula orgánica o inorgánica cargada por la existencia de un grupo sulfhidrilo que compite y desplaza un metal determinado, con carga opuesta, facilitando su eliminación vía urinaria. Un quelante ideal debe tener gran afinidad por el metal, baja toxicidad, ser capaz de competir con quelantes naturales (albúmina, hemoglobina, citocromos), alta hidrosolubilidad, rápida eliminación y que tenga similar compartimiento de distribución que el metal a quelar ${ }^{13}$. La decisión de uso de quelantes se basa en la presencia de síntomas y de los niveles en orina de mercurio metálico; se recomienda el tratamiento en casos sintomáticos o con niveles mayores a $150 \mathrm{ug} / \mathrm{g}$ de creatinina. Actualmente existen varias moléculas quelantes de uso clínico especialmente indicadas en intoxicación por metales pesados pero que podrían asociarse a efectos adversos graves:

- Ácido Calcio Disódicoetilenediamintetraacético (CaNa2-EDTA): De "amplio espectro", mala absorción digestiva, sólo se utiliza vía parenteral y puede presentar 
efectos secundarios graves asociados a la quelación de calcio, como falla renal, arritmias, tetania, pancitopenia y trastornos de coagulación, convulsiones y dificultad respiratoria ${ }^{13}$.

- D-Penicilamina: De amplio espectro, conocido por su efecto quelante de cobre en el tratamiento de la Enfermedad de Wilson. Presenta efectos secundarios graves como falla renal, leucopenia, trombocitopenia y precipitar cuadros autoinmunes ${ }^{13}$.

- BAL (2,3 Dimercaprol); DMPS (Na 2,3 Dimercaptopropanosulfonato): Ambos de amplio espectro, asociados a efectos adversos muy graves. El último no autorizado por la FDA para uso clínico ${ }^{13}$.

- Succimer (Ácido Dimercaptosuccínico): quelante hidrofílico con excelente absorción digestiva y baja frecuencia de eventos adversos (elevación transitoria de enzimas hepáticas, trombocitosis, intolerancia gástrica). Se puede utilizar en forma ambulatoria. Se describe una probable interferencia con el metabolismo del zinc y cobre y su uso en intoxicación por mercurio orgánico podría aumentar el efecto de este metal a nivel de sistema nervioso central. Se utiliza por períodos de 19 días y el control post tratamiento se realiza con niveles en orina. Para la decisión de un segundo tratamiento se utilizan los mismos criterios que para el inicio $^{13}$.

Mención especial merece el caso de ingestión de mercurio metálico ya que se debe recordar que la absorción por vía digestiva y por piel es mínima y la eliminación es rápida, por lo que no requiere de estudios de laboratorio y sólo seguimiento clínico.

Considerando la fisiopatología de la intoxicación por metales pesados, lo que se refiere a la producción de radicales libres y de peróxido de hidrógeno, el uso de antioxidantes podría tener un rol importante en el tratamiento de estos pacientes. La evidencia actual no es consistente para apoyar el uso sistemático de antioxidantes como coadyuvantes de la quelación ya que no existen estudios de seguridad en humanos ${ }^{13,14}$.

En relación al zinc, que tiene un efecto be- neficioso demostrado en el tratamiento de la intoxicación por mercurio orgánico, existen estudios que apoyan el uso de este oligoelemento como coadyuvante del tratamiento quelante ya que aumentaría la actividad del complejo enzimático glutatión-peroxidasa, con un efecto neuro y nefroprotector ${ }^{15}$.

\section{Conclusión}

Los cambios observados en la etiología de las enfermedades en la infancia, hacen necesario tener presente causas infecciosas y no infecciosas como las intoxicaciones, particularmente cuando el compromiso es multisistémico y los exámenes de apoyo diagnóstico no son concluyentes.

La intoxicación por mercurio, es un problema de salud pública importante, por ser causa de morbimortalidad y también por el desconocimiento de los potenciales efectos dañinos y la falta de difusión de las políticas claras en relación al manejo de las personas expuestas y a la disposición de lugares especiales para la eliminación de este metal. La recomendación internacional actual es retirar de todos los lugares de atención de salud y de centros educacionales, cualquier elemento que contenga mercurio. Es indispensable educar a la población general con especial énfasis en niños, padres, cuidadores, educadores y personal de salud y optimizar las políticas nacionales por un ambiente libre de mercurio.

\section{Agradecimientos}

Agradecemos a los Drs. Enrique Paris M. y Juan Carlos Ríos B. Director Médico y Director Ejecutivo del CITUC (Centro de Información Toxicológica de la Universidad Católica) y al Sr. Daniel Rojo M., Bioquímico del Laboratorio de Toxicología Laboral del Instituto de Salud Pública, por la valiosa asesoría técnica en el manejo de estos pacientes.

\section{Referencias}

1.- Allen S, Buchanan L: Mercury exposure in children: a review: Toxicology Applied Pharmacology 2004; 198 : 209-30. 
2.- Bose-O'Reilly S, McCarty KM, Steckling N, et al: Mercury Exposure and Children's Health. Curr Probl Pediatr Adolesc Health Care 2010; 40: 186-215.

3.- Ferrer A: Intoxicación por metales. Anales sis san Navarra. 2003; 26 (supl. 1): 141-53.

4.- Grandjean P, Satoh H, Murata K, et al: Adverse Effects of Methylmercury: Environmental Health Research Implications. Environmental Health Perspectives 2010; 118: 1137-45.

5.- Lee R, Middleton D, Caldwell K, et al: A Review of Events That Expose Children to Elemental Mercury in the United States. Environmental Health Perspectives 2009; 117: 871-8.

6.- Sarikaya S, Karcioglu O, Ay D, et al: Acute mercury poisoning: a case report. BMC Emergency Medicine 2010; http://www.biomedcentral.com/1471-227X/10/7, última visita 25-01-2012.

7.- Shi-Jun Li, Su-Hua Zhang, Hui-Ping Chen, et al: Mercury-Induced Membranous Nephropathy: Clinical and Pathological Features. Clin J Am Soc Nephrol 2010; 5: 439-44.

8.- Barbier O, Grégory J, Michel J, et al: Effect of Heavy Metals on, and Handling by, the Kidney. Nephron Phy- siol 2005; 99: 105-10.

9.- Alhamad T, Rooney J, Nwosu A, et al: Lessons learned from a fatal case of mercury intoxication. Int Urol Nephrol 2011 (en prensa).

10.- Al-Sinani S, Al-Rawas A, Dhawan A: Mercury as a cause of fulminant hepatic failure in a child: Case report and literature review. Clinics and Research in Hepatology and Gastroenterology 2011 (en prensa).

11.- Taber K, Hurley R: Mercury Exposure: Effects Across the Lifespan. J Neuropsychiatry Clin Neurosci 2008: 4; 384-389.

12.- Caravati E, Erdman A, Christianson G, et al: Elemental mercury exposure: An evidence-based consensus guideline for out-of-hospital management. Clinical Toxicology 2008: 46: 1-21.

13.- Swaran F, Pachauri V: Chelation in Metal Intoxication. Int. J. Environ. Res. Public Health 2010; 7: 2745-88.

14.- Ibrahim D, Froberg B, Wolf A, et al: Heavy Metal Poisoning: Clinical Presentations and Pathophysiology. Clin Lab Med 2006; 26: 67-97.

15.- Chapman L, Chan H: The influence of nutrition on methyl mercury intoxication. Environmental health perspectives 2000; 108: 29-56. 